\title{
Morbus Agglo
}

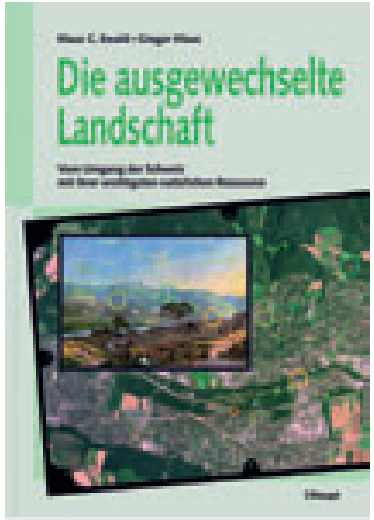

Au moyen de photographies, de graphiques et de cartes, les auteurs montrent la transformation de nos paysages.
1 Ewald KC, Klaus G. Die ausgewechselte Landschaft. Vom Umgang der Schweiz mit ihrer wichtigsten Ressource. Mit 1000 Grafiken und Fotos. Bern: Haupt-Verlag; 2009.

2 Eisinger A, Schneider M (Hrsg.). Stadtland Schweiz. Avenir Suisse. Basel: Birkhäuser-Verlag für Architekten; 2005
Les processus de destruction qui durent plus longtemps qu'une vie humaine ne sont guère pris en considération et ils le sont encore moins en l'absence de tout moyen apparent pour les contrer. Il en résulte une sorte de nihilisme thérapeutique. Personne ne se sent responsable, même quand une documentation sur l'évolution de la maladie, minutieusement établie et accessible à tous, présente clairement tous les changements intervenus. Or l'agenda politique ignore tout ce qui n'est pas gage de succès rapide. Les stratégies mentales de défense des habitants fonctionnent de manière fiable. L'idéologie de la croissance, profondément ancrée dans les mentalités, veille à relativiser le diagnostic, à le rendre éloigné de la réalité ou à le considérer comme une émanation du progrès, un produit généré par des causes externes ou le prix inévitable d'un bien-être accru. Les optimistes professionnels pensent que l'avenir remettra les choses en ordre, d'une façon ou d'une autre, et les cyniques annoncent une nouvelle glaciation qui laminera le pays. La stratégie habituelle consiste à externaliser les fautes sur des pays lointains et à accuser, par exemple, l'industrie canadienne du schiste bitumineux, la destruction des forêts amazoniennes ou la pollution des océans, au lieu de les imputer à son propre pays. Nous ne nous sentons pas responsable de la fonte des calottes polaires, même si notre pays utilise actuellement les surfaces de production de deux planètes terrestres supplémentaires rien que pour conserver notre mode de vie.

Si l'on veut élargir la mémoire individuelle par des souvenirs collectifs, on commencera par des vues et des panoramas de la Suisse réalisés par Hans Conrad Escher (1767-1823). On passera finalement à la documentation «Die ausgewechselte Landschaft», établie récemment par Klaus Ewald, professeur émérite de l'EPFZ, et par Gregor Klaus, journaliste scientifique [1]. Au moyen de photographies, de graphiques et de cartes, ce compendium montre que la transformation de nos paysages s'est accélérée au cours des 50 dernières années et que les constructions grignotent progressivement toutes les terres agricoles; Klaus Ewald et Gregor Klaus y voient une perte individuelle du sentiment d'appartenance à un coin de terre dans un pays trop bétonné, où tout ce qui n'est pas source de profit est mis de côté.

On trouvera une autre approche dans l'étude approfondie réalisée par un collectif d'architectes célèbres, lequel a examiné le territoire de cinq grandes métropoles, neuf villes et 48 agglomérations [2]. Cette étude, intitulée «Stadtland Schweiz», offre des analyses et des études de cas très intéressants concernant les structures locales, les flux de circulation, les réseaux économiques et les obstacles de politique locale. Des représentations multifonctionnelles et d'un genre nouveau renvoient l'image idéale de la campagne et l'image terrifiante de la ville envahissant la campagne au monde de l'affabulation. Au lieu de libérer les espaces intermédiaires de la pression urbaine, les autoroutes ont promu de manière involontaire la dispersion de l'habitat à une grande échelle.

Entre la métropole lémanique et le lac de Constance vivent aujourd'hui sept habitants sur dix entre des blocs d'habitation, des restes de campagne, des parcs industriels, des voies de circulation, des centres d'achats et des parcs de loisirs. Le développement transfrontalier est présenté de manière particulièrement intéressante: la région TriRhena autour de Bâle, l'Arc lémanique et ses connexions avec Lyon et Paris, le Triangolo insubrico dans la périphérie de Milan et l'Euregio autour de Constance. Ces architectes autochtones cherchent de nouveaux horizons à planifier, définissent l'urbanité et la durabilité, parlent de concentration et proposent de supprimer certaines constructions. «What could Switzerland become?» Selon leurs scénarios, la Suisse sera une plaque tournante européenne composée d'aéroports et d'un super-Zurich, le Central Park de l'Europe, un producteur d'oxygène, le château d'eau de l'Europe, un immense parc d'éoliennes et de panneaux solaires ou le pays des loisirs alpins. Dans leur étude, ils ont détecté un problème clé concernant la décentralisation encouragée par les politiques, à savoir l'absence d'organes en mesure de réaliser concrètement la planification du territoire telle qu'elle est régulièrement définie dans chaque cas en particulier. Peut-on encore stopper le processus de morcellement territorial par de nouvelles constructions sans prendre des mesures régulatrices de nature totalitaire? Des simulations sur ordinateur montrent que des terres peuvent être récupérées entre les zones urbaines aux innombrables tours d'habitation. Utopie ou réalisme?

On cite volontiers Hippocrate mais on ne lit guère ses œuvres. Dans son enseignement sur l'environnement, il estime que le physique et le caractère des habitants d'un pays s'harmonisent avec sa nature. Peut-être devrions-nous à nouveau en tenir compte, d'autant plus qu'Hippocrate ajoute que nous ne ferons pas fausse route si nous élaborons nos projets sur la base des conclusions tirées de cette affirmation.

Erhard Taverna 